\title{
BIBLIOGRAPHIE PARTIELLE DES OEUVRES
}

\section{DE PHIIOSOPHIE EN LANGUE FRANÇAISE}

\section{POUR L'ANNEE 1993.}

Alliez Eric. La Signature du monde ou Qu'est-ce que la philosophie de Deleuze et Guattari? Cerf, 1993.

Allouch, Jean. Louis Althusser, récit divan: lettre ouverte à Clément Rosset à propos de ses notes sur Louis Althusser. EPEL, 1993.

Alverny, Marie-Thérèse d'. Avicenne en Occident: recueil d'articles de Marie-Thérèse d'Alverny réunis en hommage à l'auteur. Vrin, 1993.

Anne, Chantal. L'Amour dans la pensée de Soren Kierkegaard: pseudonymie et polyonymie. L'Harmattan, 1993.

Ansaldi, Saverio. La Tentative schellingienne: un système de la liberté est-il possible? L'Harmattan, 1993.

Antoniol, Lucie. Lire Ryle aujourd'hui: aux sources de la philosophie analytique. Préf. T.S. Champlin. De Boeck-Wesmael, 1993.

Archives de philosophie, no. 55; Hobbes et Locke. Beauchesne, 1993.

Aubenque, Pierre. La Prudence chez Aristote. Nouv. éd. PUF, 1993.

-.--. Dir, Alonso Tordesillas. Dir. Aristote politique: études sur la Politique d'Aristote. PUF, 1993.

Audard, Catherine. Dir. Le Respect: de l'estime à la déférence, une question de limite. Autrement, 1993.

Bachelard, Gaston. L'Eau et les rêves: essai sur l'imagination de la matière. LGF, 1993.

Bailly, Jean-Christophe. Adieu: essai sur la mort des dieux. Ed. de l'Aube, 1993.

Belaval, Yvon. Etudes leibniziennes: de Leibniz à Hegel. Gallimard, 1993.

Bernard, Claude. Victor Cousin ou la Religion de la philosophie. Presses universitaires du Mirail-Toulouse, 1993.

Besnier, Jean-Michel. Histoire de la philosophie moderne et 
contemporaine: figures et oeuvres. Grasset, 1993.

---.. L'Humanisme déchiré. Descartes \& Cie, 1993.

Bloch, Olivier. Dir. Spinoza au XXe siècle. PUF, 1993.

Blondel, Maurice. L'Action: essai d'une critique de la vie et d'une science de la pratique, 1893. PUF, 1993.

Boituzat, François. Un Droit de mentir: Constant ou Kant? PUF, 1993. Borreil, Jean, Christine Buci-Glucksman. Ed, Genevieve Fraisse. Ed, Jacques Rancière. Ed. La Raison nomade. Préf. Jacques Rancière. Payot, 1993.

Bourg, Dominique. Dir, Antoine Lion. Dir. La Bible en philosophie: approches contemporaines. Cerf, 1993.

-.--. Dir. Les Sentiments de la nature. La Découverte, 1993.

Bourgeois, Bemard. Dir, Jacques D'Hondt. Dir. La Philosophie et la Révolution française. Vrin, 1993.

Brague, Rémi. Dir. Saint Bemard et la philosophie. PUF, 1993.

Breton, Stanislas. Matière et dispersion. J. Millon, 1993.

Brykman, Geneviève. Berkeley et le voile des mots. Vrin, 1993.

Burbage, Frank, Nathalie Chouchan, Leibniz et l'infini. PUF, 1993.

Canivez, Patrice. Le Politique et sa logique dans l'oeurre d'Eric Weil. Kimé, 1993.

Cariou, Pierre. Pascal et la casuistique. PUF, 1993.

Chalier, Catherine. Levinas: l'utopie de l'humain. Albin Michel, 1993.

-..-.. Pensées de l'étemité: Spinoza, Rosenzweig. Cerf, 1993.

Challemel-Lacour, Paul. Etudes et réflections d'un pessimiste, 1862; Un Bouddhiste contemporain en Allemagne, Arthur Schopenhauer. Fayard, 1993.

Clair, André. Kjerkegaard: penser le singulier. Cerf, 1993.

Clemens, Eric. La Fiction et l'apparaftre. Albin Michel, 1993.

Cohn, Jonas. Théorie de la dialectique: doctrine des formes philosophiques. Áge d'homme, 1993.

Comte-Sponville, André. L'Amour de la solitude: entretiens avec Patrick Vighetti, Judith Brouste, Charles Juliet. Paroles d'aube, 1993.

Conche, Marcel, Lucile Laveggi. Vivre et philosopher: réponses aux questions de Lucile Laveggi. PUF, 1993.

Crouzel, Henri. Origène et Plotin: comparaisons doctrinales. Téqui, 1993.

Cottier Georges. Histoire et connaissance de Dieu. Ed. universitaires de Fribourg, 1993.

Courcelles, Dominique de. La Parole risquée de Raymond Lulle: entre judaisme, christianisme et islam. Préf. Alain de Libera. Vrin, 1993. 
Dagognet, François. La Peau découverte. Laboratoires Delagrange, 1993.

-..-.. Réflexions sur la mesure. Encre marine, 1993.

Deleuze, Gilles. Critique et Clinique. Minuit, 1993.

Delruelle, Edouard. Le Consensus impossible: le différend entre éthique et politique chez $\mathrm{H}$. Arendt et $\mathrm{J}$. Habermas. Ousia, 1993.

Derrida, Jacques. Khôra. Galilée, 1993.

-.--. Passions. Galilée, 1993.

-.-.. Sauf le nom. Galilée, 1993.

-.--.. La Voix et le phénomène: introduction au problème du signe dans la phénoménologie de Husserl. Nouv. éd. PUF, 1993.

-.--. La Dissémination. Seuil, 1993.

-.--. Spectres de Marx: l'état de la dette, le travail du deuil et la nouvelle Internationale. Galilée, 1993.

Destutt de Tracy, Antoine. Mémoire sur la faculté de penser. Fayard, 1993.

Duchesneau, François. Leibniz et la méthode de la science. PUF, 1993.

Dumont, Jean-Paul. Ed. Eléments d'histoire de la philosophie antique. Nathan, 1993.

Elie, Maurice. Lumière, couleurs et nature: l'optique et la physique de Goethe et de la Natur-philosophie. Préf. François Dagognet. Vrin, 1993.

Fallot, Jean. Cette mort qui n'en est pas une. Presses universitaires de Lille, 1993.

Ferret, Stéphane. Le Philosophe et son scalpel: le problème de l'identité personnelle. Minuit, 1993.

Folscheid, Dominique. Dir. La Philosophie allemande: de Kant à Heidegger. PUF, 1993.

Frossard, André. L'Homme en questions. Stock, 1993.

Gaultier, Abraham. Parité de la vie et da la mort: la Réponse du médecin Gaultier. Olivier Bloch. Ed. Universitas, 1993.

Gil, Didier. Bachelard et la culture scientifique. PUF, 1993.

Gil, Femando. Traité de l'évidence. J. Millon, 1993.

Glaziou, Yves. Hobbes en France au XVIIle siècle. PUF, 1993.

Gomez-Muller, Alfredo. Dir. Penser la rencontre de deux mondes: les défis de la découverte de l'Amérique. PUF, 1993.

Goulet-Cazé, Marie-Odile. Ed, Goulet, Richard. Ed. Le Cynisme ancien et ses prolongements. PUF, 1993.

Goyard-Fabre, Simone. Montesquieu, la nature, les lois, la liberté. 
PUF, 1993.

Granarolo, Philippe. L'Individu éternel: l'expérience nietzschéenne de l'étemité. Vrin, 1993.

Greisch, Jean. Dir, Jacques Rolland. Dir. Emmanuel Lévinas: l'éthique comme philosophie. Actes du colloque de Cerisy-la-Salle, 23 août-2 sept. 1986. Cerf, 1993.

----.. Dir. Comprendre et interpréter: le paradigme herméneutique de la raison. Beauchesne, 1993.

Grimaldi, Nicolas. L'Ontologie du temps: l'attente et la rupture. PUF, 1993.

Grondin, Jean. L'Universalité de l'herméneutique. Préf. Hans Georg Gadamer. PUF, 1993.

-.-.. L'Horizon herméneutique de la pensée contemporaine. Vrin, 1993.

Guérin, Michel. L'Affectivité de la pensée; Le Concept kantien de l'analogie. Actes sud, 1993.

Guibal, Francis. Autonomie et altérité. Cerf, 1993.

Gusdorf, Georges. Le Romantisme. Payot, 1993.

Haar, Michel. Nietzsche et la métaphysique. Gallimard, 1993.

Habib, Claude. Dir, Mouchard, Claude. Dir. La Démocratie à l'oeuvre: autour de Claude Lefort. Esprit, 1993.

Hersch, Jeanne. L'Etonnement philosophique: une histoire de la philosophie. Gallimard, 1993.

Hottois, Gilbert. Simondon et la philosophie de la culture technique. De Boeck-Wesmael, 1993.

Hubeny, Alexandre. L'Action dans l'oeuvre de Hannah Arendt: du politique à l'éthique. Larousse, 1993.

Imbach, Ruedl. Ed, Marie-Thérèse Méléard. Ed. Philosophes médiévaux des XIIle et XIVe siècles. UGE, 1993.

Jeu, Bemard. Le Sportif, le philosophe, le dirigeant. Université de Lille III, 1993.

Klibansky, Raymond. Dir. David Pears. Dir. La Philosophie en Europe. Gallimard, 1993.

Knee, Philip. Qui perd gagne: essai sur Sartre. Presses de l'Université de Laval, 1993.

Kobayashi, Michio. La Philosophie naturelle de Descartes. Pref. Jules Vuillemin. Vrin, 1993.

Lachelier, Jules. Du fondement de l'induction. Fayard, 1993.

Landsberg, Paul-Louis. Essai sur l'expérience de la mort; Le Problème moral du suicide. Préf. Jean Lacrois; postf. Olivier Mongin. Seuil, 1993. 
Lanteigne, Josette. La Question du jugement. L'Harmattan, 1993.

Lardreau, Guy. La Véracité: essai d'une philosophie négative. Verdier, 1993.

Largeault, Jean. Intuition et Intuitionisme. Vrin, 1993.

----. La Logique. PUF, 1993.

Laurent, Alain. Histoire de l'individualisme. PUF, 1993.

Laux, Henri. Imagination et religion chez Spinoza: la potentia dans l'histoire. Vrin, 1993.

Lavelle, Louis. La Conscience de soi. C. de Bartillat, 1993.

Lazarus, Sylvain. Dir. Politique et philosophie dans l'oeuvre de Louis Althusser. Colloque, Université de Paris VIII, 29-30 mars 1991. PUF, 1993.

Leclerc, Marc. La Destinée humaine: pour un discernement philosophique. Culture et Vérité, 1993.

Lecourt, Dominique. Dir, Françoise Duroux. Dir. Canguilhem, philosophe et historien des sciences. Albin Michel, 1993.

----. A quoi sert donc la philosophie? PUF, 1993.

Le Diraison, Serge, Eric Zemik. Le Corps des philosophes. PUF, 1993.

Léger, François. Monsieur Taine. Critérion, 1993.

Lenain, Thierry. Pour une critique de la raison ludique: essai sur la problématique nietzschéenne. Vrin, 1993.

Lequier, Jules, André Clair. Ed. La Recherche d'une première vérité. Préf. Charles Renouvier. PUF, 1993.

Levinas, Emmanuel. Dieu, la mort et le temps. Grasset, 1993.

---.-. Entre nous: essais sur le penser de l'autre. LGF, 1993.

Libera, Alain de. La Philosophie médiévale. PUF, 1993.

Libis, Jean. L'Eau et la mort. Ed. universitaires de Dijon, 1993.

Longuenesse, Béatrice. Kant et le pouvoir de juger. PUF, 1993.

Losurdo, Domenico. Autocensure et compromis dans la pensée politique de Kant. Presses universitaires de Lille, 1993.

Lyotard, Jean-François. Le Postmodeme expliqué aux enfants: correspondance, 1983-1985. LGF, 1993.

Marin, Louis. Des pouvoirs de l'image. Seuil, 1993.

Marion, Jean-Luc. Sur l'ontologie grise de Descartes: science cartésienne et savoir aristotélicien dans les Regulae. उe éd. Vrin, 1993.

Martin, Jean-Clet. Variations: la philosophie de Gilles Deleuze. Payot, 1993.

Mascolo, Dyonis. Haine de la philosophie: Heidegger comme modèle, bassesse et profondeur. J-M. Place, 1993.

Mattel, Jean-François. Pythagore et les pythagoriciens. PUF, 1993. 
Maury, Liane. Les Emotions de Darwin à Freud. PUF, 1993.

Mengue, Philippe. Gilles Deleuze. Belfond, 1993.

Michel, Romain. Du Dieu des philosophes au Dieu des chrétiens: aude-là du catéchisme. Quinquet, 1993.

Millet, Louis, Isabelle Mourral. Petite encyclopédie de philosophie. Ed. universitaires, 1993.

Millon-Delsol, Chantal. L'Esprit européen. Mame, 1993.

Missa, Jean-Noël. L'Esprit-cerveau: la philosophie de l'esprit à la lumière des neurosciences. Préf. Claude Debru. Vrin, 1993.

Monk, Ray. Ludwig Wittgenstein: le devoir du génie. O. Jacob, 1993.

Morel, Pierre. Ed. Démocrite et l'atomisme ancien: les textes et les thèmes fondamentaux de la philosophie et de la science. Presses-Pocket, 1993.

Muglioni, Jean-Michel. La Philosophie de l'histoire de Kant: Qu'est-ce que l'homme? PUF, 1993.

Mulligan, Kevin. Dir. Wittgenstein analysé. Préf. Jean-Pierre Leyvraz. J. Chambon, 1993.

Nancy, Jean-Luc. Le Sens du monde. Galilée, 1993.

Natan. De la morale fonadmentale aux éthiques appliquées. Jafo, 1993.

Ogien, Ruwen. Un Portrait logique et moral de la haine. Eclat, 1993.

Parrochia, Daniel. La Raison systématique: essaï d'une morphologie des systèmes philosophiques. Vrin, 1993.

-.--.. Philosophie des réseaux. PUF, 1993.

Pasqua, Hervé. Introduction à la lecture de Etre et temps de Martin Heidegger. Age d'homme, 1993.

Pauli, Evaldo. Les Sentiers sinueux de la connaissance: traité de gnoséologie sur le doute et la certitude. Academia, 1993.

Philonenko, Alexis. Lecture de la phénoménologie de Hegel: Préface, Introduction. Vrin, 1993.

Planty-Bonjour, Guy. Le Projet hégélien. Vrin, 1993.

Poulin, Jacques. La Loi de vérité ou la Logique philosophique du jugement. Albin Michel, 1993.

-.-.-. La Neutralisation du jugement ou la Critique pragmatique de la raison politique. L'Harmattan, 1993.

Quéran, Odile. Ed, Denis Trarieux. Ed. Les Discours du corps: une anthologie. Presse-Pocket, 1993.

Quéré, Louis. La Théorie de l'action: le sujet pratique en débat. Ed. du CNRS, 1993.

Quilliot, Roland. La Liberté. PUF, 1993.

Quiniou, Yvon. Nietzsche ou l'Impossible Immoralisme. Kimé, 1993.

Radrizzani, Ives. Vers la fondation de l'intersubjectivité chez Ficht: des 
Principes à la Nova Methodo. Vrin, 1993.

Renaut, Alain. Sartre, le demier philosophe. Grasset, 1993.

Rivenc, François. Recherches sur l'universalisme logique: Russell et Camap. Payot, 1993.

Rosset, Clément. Le Réel et son double: essai sur l'illusion. Gallimard, 1993.

-.--.. Logique du pire: éléments pour une philosophie tragique. Nouv. éd. PUF, 1993.

Rousseau, Jean-Jacques, Kintzler, Catherine. Ed. Essai sur l'origine des langues. Flammarion, 1993.

Roux, Georges. Le Temps des plénitudes. Age d'homme, 1993.

Roviello, Anne-Marie. Dir, Weyembergh, Maurice. Dir. Hannah Arendt et la modemité. Vrin, 1993.

Russ, Jacqueline. Dir. Histoire de la philosophie. Armand Colin, 1993.

Saint-Girons, Baldine. Fiat lux. Quai Voltaire, 1993.

Sauvanet, Pierre. L'Insu. Ed. du Cavalier vert, 1993.

Schaal, Jean-François. Le Corps: cours préparation HEC. EllipsesMarketing, 1993.

Schérer, René. Zeus hospitalier: éloge de l'hospitalité. Essai philosophique. Armand Colin, 1993.

Schwarz Theodor. Irrationalisme et humanisme. Age d'homme, 1993.

Semin, André. Auguste Comte prophète du XIXe siècle: sa vie, son ceuvre et son actualité: Auguste Comte et nous. Albatros, 1993.

Simon, René. Ethique de la responsabilité. Cerf, 1993.

Skirbekk, Gunnar. Rationalité et modernité. L'Harmattan, 1993.

Tenace, Michelina. La Beauté, unité spirituelle: dans les écrits esthétiques de Vladimir Soloviev. Préf. Olivier Clément. Fates, 1993.

Thiry, Philippe. Notions de Logique. De Boeck-Wesmael, 1993.

Tiercelin, Claudine. La Pensée-signe: étude sur C.S. Peirce. J. Chambon, 1993.

Ucciani, Louis. De l'Ironie socratique à la dérision cynique: éléments pour une critique par les formes exclues. Belles lettres, 1993.

Vadée, Michel. Marx, penseur du possible. Méridiens-Klincksieck, 1993.

Vergely, Bertrand. Ed. La Philosophie. Larousse, 1993.

Vergotte, Henri-Bernard. Lectures philosophiques de Soren Kierkegaard: Kierkegaard chez ses contemporains danois. PUF, 1993. 
Vincent, Bernard. Dir. Thomas Paine ou la République sans frontières. Préf. Madeleine Rebérioux; postf. Michel Vovelle. Presses universitaires de Nancy, 1993.

Voelke, André-Jean. La Philosophie comme thérapie de l'âme: études de philosophie hellénistique. Préf. Pierre Hadot. Cerf: Ed. universitaires de Fribourg, 1993.

Wunenburger, Jean-Jacques. Questions d'ethique. PUF, 1993. 